\title{
Spin cat states in ferromagnetic insulators
}

\author{
Sanchar Sharma $\odot,{ }^{1}$ Victor A. S. V. Bittencourt, ${ }^{1}$ Alexy D. Karenowska, ${ }^{2}$ and Silvia Viola Kusminskiy ${ }^{1,3}$ \\ ${ }^{1}$ Max Planck Institute for the Science of Light, 91058 Erlangen, Germany \\ ${ }^{2}$ Clarendon Laboratory, Department of Physics, University of Oxford, Oxford OX1 3PU, United Kingdom \\ ${ }^{3}$ Department of Physics, University Erlangen-Nürnberg, 91058 Erlangen, Germany
}

(Received 17 September 2020; revised 4 December 2020; accepted 10 February 2021; published 4 March 2021)

\begin{abstract}
Generating nonclassical states in macroscopic systems is a long-standing challenge. A promising platform in the context of this quest are novel hybrid systems based on magnetic dielectrics, where photons can couple strongly and coherently to magnetic excitations, although a nonclassical state therein is yet to be observed. We propose a scheme to generate a magnetization cat state, i.e., a quantum superposition of two distinct magnetization directions, using a conventional setup of a macroscopic ferromagnet in a microwave cavity. Our scheme uses the ground state of an ellipsoid shaped magnet, which displays anisotropic quantum fluctuations akin to a squeezed vacuum. The magnetization collapses to a cat state by either a single photon or a parity measurement of the microwave cavity state. We find that a cat state with two components separated by $\sim 5 \hbar$ is feasible and briefly discuss potential experimental setups that can achieve it.
\end{abstract}

DOI: 10.1103/PhysRevB.103.L100403

Introduction. Superposition is a cornerstone of quantum theory, a paradigmatic example of which is a "cat state" referring to, loosely speaking, a system which exists in a quantum superposition of two quasiclassical states. Besides their important historical link to Schrödinger's famous gedanken experiment, their insensitivity to particle loss noise [1] means that cat states find useful application as carriers of information (qubits) in quantum computation [2-4] or as sensors in quantum metrological tasks [5-8]. The robustness of a cat state increases with its size, i.e., how "distinct" the two quasiclassical components are. Experimental realizations of cat states include photon states at optical [9-11] and microwave [12] frequencies with a size of up to 3 and 100 photons, respectively, and a spin-state with size $\sim 2 \hbar$ composed of $\sim 3000$ atoms [13]. However, nonclassical states are notoriously difficult to generate in macroscopic systems due to the lack of long-enough coherence lengths. Considerable advances on this front were obtained in optomechanical systems (in which light couples to acoustic excitations [14]) [15-18]; however, cat states have not yet been realized in this platform [19,20].

Over the past few years, a new kind of hybrid quantum system has emerged as a promising platform for quantum applications, where photons are coupled coherently to magnetic excitations (magnons) in macroscopic magnetic materials [21]. The dielectric ferrimagnet yttrium iron garnet (YIG) is the material of choice in current experiments, owing partly to its extremely low magnetic dissipation [22]. Coherent and

Published by the American Physical Society under the terms of the Creative Commons Attribution 4.0 International license. Further distribution of this work must maintain attribution to the author(s) and the published article's title, journal citation, and DOI. Open access publication funded by the Max Planck Society. strong magnon-microwave coupling using sub-mm spheres of YIG was realized [23-25] and used to mediate the coupling between magnons and superconducting qubits [26]. Magnons also coherently couple to optical photons [23,27-30], phonons [31,32], and electrons [33-35], pointing to the possibility of magnon-based quantum transducers [36,37]. Moreover, a Bose-Einstein condensate of magnons showing macroscopic coherence was demonstrated in YIG thin films [38,39]. These developments together with the recent demonstration of single-magnon detection in YIG spheres [40,41], has opened prospects for studying and manipulating microwave magnetic excitations in a quantum coherent manner. Creating nonclassical states of the magnetization is crucial for future applications in what is denominated "quantum magnonics" [21]. Theoretical proposals include all-optical heralding of magnon Fock states [42] and generation of entangled states [43-45].

Here we propose a setup to prepare a cat state of a macroscopic number of spins $\left(>10^{18}\right)$ that can be achieved by employing state-of-the-art microwave cavities with an embedded magnet of anisotropic shape, see Fig. 1(a). The protocol relies on the anisotropy of the magnet enforcing a magnetic ground state analogous to the squeezed vacuum in quantum optics, plus the concomitant entangled spin-photon ground state when the magnet is coupled to the cavity. In contrast with the case of photons, squeezing in magnetization does not require an external excitation, so does not decay [46,47]. We show that in a YIG sample, cats with a size $\sim 5 \hbar$ are feasible.

Model. A well-established protocol to generate cat states in quantum optics is to add a photon to a squeezed optical vacuum $[9,48,49]$. In order to accomplish this analogously in a ferromagnet, we first require a squeezed magnetization state [46], i.e., a minimum uncertainty state with anisotropic zero-point fluctuations. This is realized via the ground state of a magnet with an anisotropic shape, such as an ellipsoid. Notably, the degree of squeezing of the magnetic ground state 
(a)
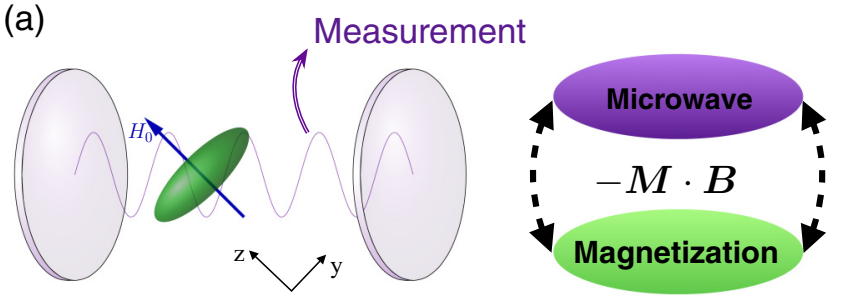

(b)

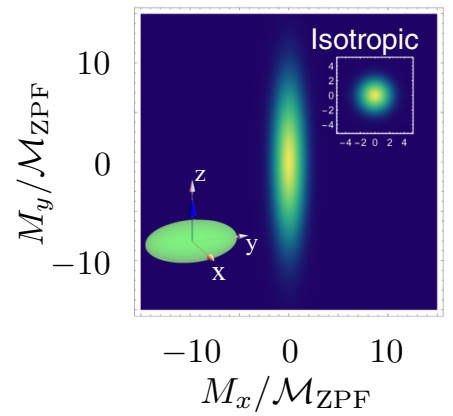

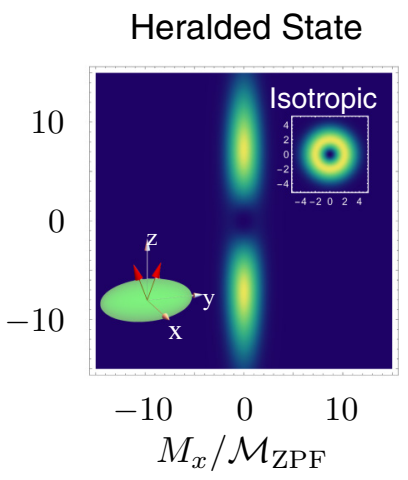

FIG. 1. (a) Setup: A ferromagnetic ellipsoid (green) with magnetization $\boldsymbol{M}$ couples to microwaves in a cavity via Zeeman coupling to the cavity magnetic field $\boldsymbol{B}$. An external applied field $H_{0}$ saturates the magnetization along one of the short axes $(\| \boldsymbol{z})$. (b) Probability density [Husimi $Q$ function, Eq. (9)] of the quasiclassical magnetization's state as a function of its components in units of the isotropic zero point fluctuations $\mathcal{M}_{\mathrm{ZPF}}$ Eq. (1). Ground state: The squeezed magnetization vacuum [see Eq. (10)] with anisotropic fluctuations. The inset shows the nonsqueezed (isotropic) case, valid for spherical magnets. Heralded state: The magnetization state after detecting a microwave photon [see Eq. (20)] showing features of a cat state. Such features are absent when there is no squeezing (inset). The magnetization is in a pure state after heralding, so these features cannot be due to a classical probability distribution.

is tunable by an external magnetic field. The second step is to add an excitation, i.e., to "flip" on average one spin which is delocalized in space, see Fig. 1(b). We show that this can be achieved by coupling the magnetization to a microwave cavity [23-25] and performing a measurement of the latter. For low-enough temperatures either a single-photon measurement [50,51] or a parity measurement can be employed [52]. We discuss these steps in detail below.

The proposed setup is shown in Fig. 1(a) where a ferromagnetic ellipsoid is kept inside a microwave cavity. The magnet is assumed to be slender and prolate, i.e., $L_{y} \gg L_{x}=$ $L_{z}$, where $L_{i}$ is the length in $i$ th direction. In the absence of external magnetic fields, the magnetization would align with the longest axis. A sufficiently large field, here $H_{0} z$, aligns the magnetization to $z$ with quantum (zero-point) fluctuations largely along $\boldsymbol{y}$ [46], see Fig. 1(b). For comparison, spherical magnets have isotropic zero point fluctuations $\mathcal{M}_{\mathrm{ZPF}}$ given by

$$
\mathcal{M}_{\mathrm{ZPF}}^{2}=\frac{\gamma \hbar M_{s}}{2 V}
$$

where $V$ is the volume of the magnet and $\gamma$ is absolute value of the gyromagnetic ratio. The fluctuations are a consequence of the Heisenberg uncertainty relations, embodied

by the spin commutation relations $\left[S_{x}, S_{y}\right]=i \hbar S_{z}$ (note that $\boldsymbol{M}=\gamma \boldsymbol{S} / V$, where $\boldsymbol{S}$ is the macrospin). Flipping a (delocalized) spin pushes the magnetization away from the origin. In the presence of shape anisotropy, the resulting state has the characteristic features of a cat state involving a superposition of two sufficiently distinct semiclassical states, see Fig. 1(b).

In macrospin limit, the classical Hamiltonian density for the magnetization is

$$
\mathcal{H}_{\text {mag }}=\frac{\mu_{0}}{2} \boldsymbol{M} \tilde{N} \boldsymbol{M}-\mu_{0} M_{z} H_{0},
$$

where $\boldsymbol{M}$ is the total magnetization and $\tilde{N}$ is the demagnetization tensor. The magnitude $|\boldsymbol{M}|=M_{s}$ is a constant of motion [53], where $M_{s}$ is the saturation magnetization. For spheroids, $\tilde{N}$ is diagonal with $N_{x}=N_{z}=N_{T}$ and $N_{y}=1-2 N_{T}[53,54]$. We assume a sufficiently large magnetic field, $H_{0}>M_{s} / 2$, such that the classical ground state is $\boldsymbol{M}=M_{s} z$. Due to a difference in energy cost, the fluctuations in $M_{y}$ are larger than that in $M_{x}$, leading to a squeezed vacuum [46]. We model the quantum fluctuations using the Holstein-Primakoff approximation [53,55],

$$
\frac{M_{x}-i M_{y}}{2 \mathcal{M}_{\mathrm{ZPF}}} \rightarrow \hat{s}
$$

valid for $\left|M_{x, y}\right| \ll M_{z}$ with $\mathcal{M}_{\mathrm{ZPF}}$ defined in Eq. (1). $M_{z}$ is found by the constraint $|\boldsymbol{M}|=M_{s}$. Using Eq. (3) and retaining only quadratic terms in $\hat{s}$, the magnetic Hamiltonian density (2) integrates to

$$
\frac{\hat{H}_{\mathrm{mag}}}{\hbar}=\omega_{0} \hat{s}^{\dagger} \hat{s}+\frac{\omega_{s}}{2}\left(\hat{s}^{2}+\hat{s}^{\dagger 2}\right)
$$

where

$$
\omega_{s}=\left(3 N_{T}-1\right) \frac{\gamma \mu_{0} M_{s}}{2}, \quad \omega_{0}=\gamma \mu_{0} H_{0}-\omega_{s} .
$$

The bosonic operator $\hat{s}$ flips a spin from $+z$ to $-z$, satisfies the canonical commutation relation $\left[\hat{s}, \hat{s}^{\dagger}\right]=1$, and annihilates the classical ground state corresponding to all spins pointing along $-z$ (the spins are antiparallel to the magnetization), $\hat{s}|0\rangle=0$. For spheres, $N_{T}=1 / 3$ implying $\omega_{s}=0$ and hence $\hat{s}\left(\hat{s}^{\dagger}\right)$ is the annihilation (creation) operator of elementary excitations of the magnet, i.e., magnons. Below, we consider the case of a slender prolate spheroid with $N_{T} \approx 1 / 2$ where the ground state is not $|0\rangle$ because of the term $\propto\left(\hat{s}^{2}+\hat{s}^{\dagger 2}\right)$.

Squeezed magnetic vacuum. The Hamiltonian (4) diagonalizes to $\hat{H}_{\text {mag }}=\hbar \omega_{m} \hat{m}^{\dagger} \hat{m}$ by a Bogoliubov transformation $\hat{m}=\cosh r_{g} \hat{s}+\sinh r_{g} \hat{s}^{\dagger}$, with

$$
\omega_{m}=\sqrt{\omega_{0}^{2}-\omega_{s}^{2}}=\gamma \mu_{0} \sqrt{H_{0}\left(H_{0}-\frac{M_{s}}{2}\right)} .
$$

The parameter $r_{g}$ characterizes the degree of squeezing and is given by

$$
e^{r_{g}}=\sqrt{\frac{\omega_{0}+\omega_{s}}{\omega_{m}}}=\left(1-\frac{M_{s}}{2 H_{0}}\right)^{-1 / 4} .
$$

The ground state of $\hat{H}_{\text {mag }}$, defined via $\hat{m}|g\rangle=0$, is given by $[46,56]|g\rangle=\mathbb{S}_{\hat{s}}\left(r_{g}\right)|0\rangle$, where

$$
\mathbb{S}_{\hat{x}}(r)=\exp \left[\frac{r\left(\hat{x}^{2}-\hat{x}^{\dagger 2}\right)}{2}\right]
$$


is the squeezing operator. It has a nonzero number of "flipped spins", $\left\langle g\left|\hat{s}^{\dagger} \hat{s}\right| g\right\rangle=\sinh ^{2} r_{g}$.

The characteristics of the ground state $|g\rangle$ can be visualized in terms of semiclassical magnetization states $|\alpha\rangle$, defined as the ones with an average magnetization $M_{x}-i M_{y}=2 \mathcal{M}_{\mathrm{ZPF}} \alpha$ and minimum fluctuations [cf. Eq. (3)]. These are given by coherent states [57], satisfying $\hat{s}|\alpha\rangle=\alpha|\alpha\rangle$, defined by $|\alpha\rangle=\hat{D}(\alpha)|0\rangle$ where the displacement operator is $\hat{D}(\beta)=$ $\exp \left[\beta \hat{s}^{\dagger}-\beta^{*} \hat{s}\right]$. For a general state $|\psi\rangle$, the Husimi $Q$ function

$$
Q(\alpha,|\psi\rangle)=\frac{1}{\pi}|\langle\alpha \mid \psi\rangle|^{2}
$$

can be interpreted as the probability density of $|\psi\rangle$ being near the semiclassical state $|\alpha\rangle$. For the ground state $|g\rangle$, defined above, we find

$$
Q(\alpha,|g\rangle)=\frac{1}{\pi \cosh r_{g}} \exp \left[-\frac{\alpha_{R}^{2} e^{r_{g}}+\alpha_{I}^{2} e^{-r_{g}}}{\cosh r_{g}}\right],
$$

where $\alpha=\alpha_{R}+i \alpha_{I}$. This is shown in Fig. 1(a) demonstrating that fluctuations in $M_{y}$ are larger than that in $M_{x}$ by a factor $e^{2 r_{g}}$, indicating a squeezed vacuum. For $H_{0} \gg M_{s} / 2, r_{g} \approx 0$ and the fluctuations are isotropic as expected. The degree of squeezing, Eq. (7), becomes arbitrarily high as $H_{0} \rightarrow M_{s} / 2$. In this limit $\omega_{m} \rightarrow 0$ and the system goes toward an instability signaling a significant change in classical ground state and a consequent failure of the linearization used in Eq. (3). In practice, however, the frequency $\omega_{m}$ is bounded by the temperature limitations of the refrigerator. Considering an experimentally feasible limit of $T>5 \mathrm{mK}$, corresponding to a magnon's frequency of $\omega_{m}>2 \pi \times 100 \mathrm{MHz}$, we obtain $e^{r_{g}}<5$, corresponding to a $<80 \%$ squeezing, where we used $\gamma \mu_{0} M_{s}=2 \pi \times 5 \mathrm{GHz}$ for YIG [53]. This requires a magnetic field $H_{0}$ slightly higher than $M_{s} / 2=70 \mathrm{kA} \mathrm{m}^{-1}$.

Coupling to a microwave cavity. The classical Hamiltonian density for a hollow cavity reads

$$
\mathcal{H}_{\text {cav }}=\frac{\epsilon_{0}|\boldsymbol{E}(\boldsymbol{r})|^{2}}{2}+\frac{|\boldsymbol{B}(\boldsymbol{r})|^{2}}{2 \mu_{0}} .
$$

We assume that the cavity consists of a single mode since the generalization does not change the qualitative features. The quantization $\boldsymbol{B}(\boldsymbol{r}) \rightarrow \boldsymbol{B}_{0}(\boldsymbol{r}) \hat{a}+\boldsymbol{B}_{0}^{*}(\boldsymbol{r}) \hat{a}^{\dagger}$, where $\boldsymbol{B}_{0}$ is the mode profile and $\hat{a}$ is the annihilation operator of the cavity mode [and analogously for $\boldsymbol{E}(\boldsymbol{r})$ ], diagonalizes the cavity Hamiltonian $\hat{H}_{\text {cav }}=\hbar \omega_{a} \hat{a}^{\dagger} \hat{a}$. The magnetization couples to microwave fields via $\mathcal{H}_{\text {coup }}=-\boldsymbol{M} \cdot \boldsymbol{B}(\boldsymbol{r})$ inside the magnet and $\mathcal{H}_{\text {coup }}=0$ outside. For magnets much smaller than microwave's wavelength $\sim \mathrm{cm}$, the magnetic field is nearly constant inside the magnet. From Eq. (3), we get

$$
\frac{\hat{H}_{\text {coup }}}{\hbar}=\left(g_{-}^{*} \hat{s} \hat{a}^{\dagger}+g_{-} \hat{s}^{\dagger} \hat{a}\right)+\left(g_{+}^{*} \hat{s}^{\dagger} \hat{a}^{\dagger}+g_{+} \hat{s} \hat{a}\right),
$$

where we ignored a tertiary term $\propto \hat{s}^{\dagger} \hat{s} \hat{a}$ with a coefficient smaller than $g_{ \pm}$by a factor $\sim \mathcal{M}_{\mathrm{ZPF}} / M_{s}$. The beam-splitter $\left(g_{-}\right)$and parametric-amplifier $\left(g_{+}\right)$coupling strengths are given by

$$
g_{ \pm}=-V \mathcal{M}_{\mathrm{ZPF}} B_{0 \pm}\left(\boldsymbol{r}_{\text {magnet }}\right),
$$

where $B_{0 \pm}=B_{0 x} \pm i B_{0 y}$ and $\boldsymbol{r}_{\text {magnet }}$ is the position of the magnet inside the cavity. Below, we consider circularly polarized photons with $B_{0+}=0$ (hence $g_{+}=0$ ) and define $g_{-} \equiv g$. The results below are not significantly affected by polarization. By changing the phase of photons, if necessary, we assume $g>0$. Depending on the experimental setup, $g$ is tunable up to a large fraction of the cavity's frequency $[58,59]$.

Results. The total Hamiltonian is

$$
\frac{\hat{H}}{\hbar}=\omega_{0} \hat{s}^{\dagger} \hat{s}+\frac{\omega_{s}}{2}\left(\hat{s}^{2}+\hat{s}^{\dagger 2}\right)+\omega_{a} \hat{a}^{\dagger} \hat{a}+g\left(\hat{s} \hat{a}^{\dagger}+\hat{s}^{\dagger} \hat{a}\right) .
$$

Throughout what follows we assume $\omega_{m}, g \ll \omega_{s}, \omega_{a}$ implying a large spin squeezing [cf. Eqs. (6,7)], whereas the exact results are given in Sec. II in the Supplemental Material [60]. The Hamiltonian has two eigenmodes at frequencies $\left\{\Omega_{m}, \Omega_{a}\right\}$, where $\Omega_{a} \approx \omega_{a}$ and

$$
\Omega_{m} \approx \sqrt{\omega_{m}^{2}-\frac{2 g^{2} \omega_{s}}{\omega_{a}}}
$$

is dispersively shifted from the bare magnon's frequency $\omega_{m}$. For large couplings, $g>\omega_{a}\left(\omega_{0}-\omega_{s}\right)$, the system becomes unstable. In the ground state $|\mathrm{vac}\rangle\rangle$, the photons and spins are squeezed and correlated as opposed to the classical ground state $|0\rangle\rangle$ (defined by $\hat{s}|0\rangle\rangle=\hat{a}|0\rangle\rangle=0$ ) [60]. In the dispersive limit $\omega_{m}, g \ll \omega_{a}$, the number of photons is small, so we expand

$$
\left.|\mathrm{vac}\rangle\rangle \approx\left(1+\sqrt{P} \frac{\hat{s}^{\dagger} \hat{a}^{\dagger}}{\cosh r_{e}}\right) \mathbb{S}_{\hat{s}}\left(r_{e}\right)|0\rangle\right\rangle,
$$

where $\mathbb{S}$ is defined in Eq. (8). The component with no photons represents a squeezed vacuum of spins with effective magnetization squeezing $r_{e}$ given by

$$
e^{2 r_{e}} \approx \frac{2 \omega_{s}}{\Omega_{m}} .
$$

For $g=0$, this reduces to $r_{e}=r_{g}$. The probability of finding the cavity in a single photon state is

$$
P \approx \frac{g^{2} \omega_{s}}{2 \Omega_{m} \omega_{a}^{2}} .
$$

The ground state consists of a nonzero number of spin-flips, $\left\langle\hat{s}^{\dagger} \hat{s}\right\rangle \approx e^{2 r_{e}} / 4$, and photons, $\left\langle\hat{a}^{\dagger} \hat{a}\right\rangle \approx P$, where the averages are taken with respect to $|\mathrm{vac}\rangle\rangle$. In the limit of infinite squeezing, $\Omega_{m} \rightarrow 0$, the number of spin flips (and photons) diverges. For more realistic squeezing ( $e^{r_{e}} \sim 5$ see discussion above) and $g \ll \omega_{a}$, the number of photons in $\left.|\mathrm{vac}\rangle\right\rangle$ is small and Eq. (16) holds.

Projecting the ground state $|v a c\rangle\rangle$ to a single-photon state, we find that the magnetization collapses to the state

$$
|C\rangle=\frac{1}{\cosh r_{e}} \hat{s}^{\dagger} \mathbb{S}_{\hat{s}}\left(r_{e}\right)|0\rangle,
$$

which corresponds to flipping a spin from the squeezed vacuum $\mathbb{S}_{\hat{s}}\left(r_{e}\right)|0\rangle$. To visualize $|C\rangle$, we consider the probability density defined by Husimi $Q$ function Eq. (9),

$$
Q(\alpha,|C\rangle)=\frac{\alpha_{R}^{2}+\alpha_{I}^{2}}{\pi \cosh ^{3} r_{e}} \exp \left[-\frac{\alpha_{R}^{2} e^{r_{e}}+\alpha_{I}^{2} e^{-r_{e}}}{\cosh r_{e}}\right],
$$

for $\alpha=\alpha_{R}+i \alpha_{I}$. This is plotted in Fig. 1(b) showing two regions of high probability. Specifically, we separate the upper 


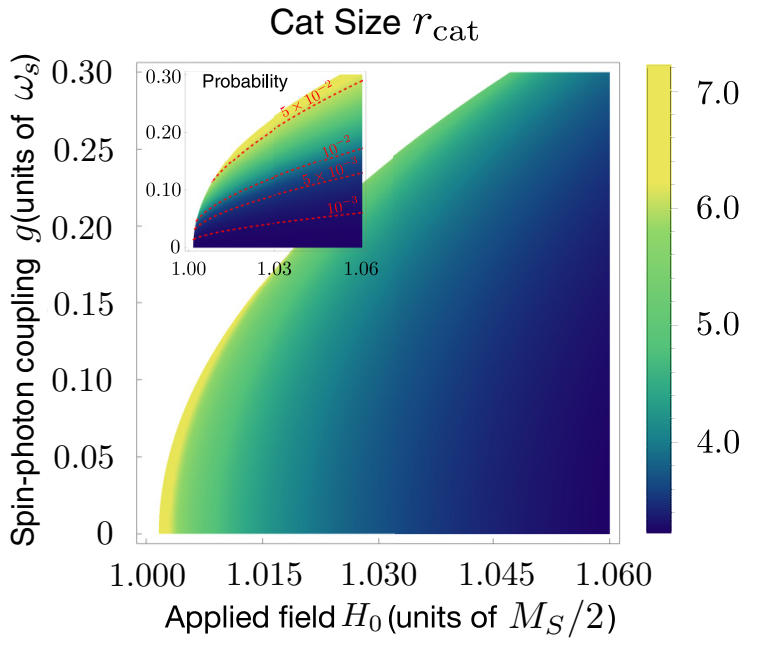

FIG. 2. Cat size $r_{\text {cat }}$ and probability (inset, logarithmic scale) of finding the cavity in a single-photon state $P$ as a function of applied field $H_{0}$ and spin-photon coupling $g$ for $\omega_{s}=\omega_{a}=2 \pi \times 1.25 \mathrm{GHz}$ (valid for YIG [53]). The white region contains the parameter space where either the system is unstable or the magnon's frequency is too low, $\Omega_{m}<2 \pi \times 100 \mathrm{MHz}$.

and lower lobes, $|C\rangle \propto\left|C_{+}\right\rangle-\left|C_{-}\right\rangle$, where

$$
\left|C_{ \pm}\right\rangle \propto \sum_{m=0}^{\infty} \frac{( \pm 1)^{m} \sqrt{m !}}{\Gamma(m / 2+1 / 2)}\left(\frac{-\tanh r_{e}}{2}\right)^{\frac{m-1}{2}}|m\rangle,
$$

with $\Gamma$ being the gamma function. Explicit calculations show

$$
\left\langle C_{-} \mid C_{+}\right\rangle \approx \frac{8 e^{-3 r_{e}}}{3 \pi},
$$

where we ignored terms higher order in $e^{-r_{e}}$, so the two components are nearly orthogonal for $e^{r_{e}} \sim 5$, showing that $|C\rangle$ is a superposition of two nearly-orthogonal semiclassical states, i.e., a cat state. $Q(\alpha,|C\rangle)$ has two peaks at $\alpha= \pm i r_{\text {cat }} / 2$ with cat size

$$
r_{\mathrm{cat}}=\sqrt{2\left(e^{2 r_{e}}+1\right)} \approx 2 \sqrt{\frac{\omega_{s}}{\Omega_{m}}},
$$

where the approximation holds for $\omega_{s}, \omega_{a} \gg \Omega_{m} \gg g^{2} / \omega_{a}$ [61]. The maxima are found by noticing that, for a fixed $|\alpha|$, $Q$ is maximized at $\alpha$ being purely imaginary. Thus, we put $\alpha_{R}=0$ and differentiate with respect to $\alpha_{I}$ to find the peaks.

In Fig. 2, we plot the cat size $r_{\text {cat }}$ and heralding probability $P$ as a function of external magnetic field $H_{0}$ (which controls the parameter $r_{e}$ via its dependence on $\omega_{m}$ ) and spinphoton coupling $g$. The plots are generated using the exact expressions given in the Supplemental Material [60] [Eqs. (36) and (37)], instead of the approximate ones discussed here. We have only plotted for the parameter regime where $\Omega_{m}>$ $2 \pi \times 100 \mathrm{MHz}$, corresponding to a cryogenic temperature $T=5 \mathrm{mK}$, as lower frequencies put a harsh requirement on refrigerators. There is a wide range of parameters where $r_{\text {cat }} \sim$ 5-7 with heralding probability $P>0.01$. The plots show a trade-off: The maximum achievable probability increases with increasing $g$, as expected, while cat size decreases. A larger coupling $g$ puts a lower limit on the bare magnon's frequency [cf. Eq. (15)] and consequently an upper limit on magnetization squeezing [cf. Eq. (7)].

Single photon detection in microwave cavities typically involves long protocols and significant errors [50,51]. However, in the limit of small photon numbers, zero and one photon states can also be distinguished by their parity, which can be measured with a high accuracy by coupling the cavity to a qubit [52]. Projecting onto the odd-parity photon state, the density matrix of the spins is given by a partial trace over the photons

$$
\hat{\rho}_{p}=\operatorname{Tr}_{\hat{a}}\left[\frac{I-(-1)^{\hat{a}^{\dagger} \hat{a}}}{2}|\mathrm{vac}\rangle\right\rangle\langle\langle\mathrm{vac}|] .
$$

To compare $\hat{\rho}_{p}$ with $|C\rangle$, we use the fidelity measure [62,63] $F=\left\langle C\left|\hat{\rho}_{p}\right| C\right\rangle$ interpreted as the probability of finding the magnetization in the state $|C\rangle$ (19). When $\omega_{s}, \omega_{a} \gg \Omega_{m} \gg$ $g^{2} / \omega_{a}$, we find $1-F \sim P^{2}$. As $P<0.1$ (see Fig. 2), we get a high fidelity $F>0.99$, implying that our results can be used with parity measurements as well.

Experimental considerations. A range of different approaches can be envisaged for experimental design of the above setup. The maximum dimension of the YIG sample employed is set by the maximum length over which coherence can be maintained which, in high-purity monocrystalline YIG, is expected to be over $100 \mu \mathrm{m}[40,41,64]$. In order to perform a microwave measurement, the sample must be installed in a microwave cavity coupled to a Josephson junction-based qubit. Here, the main decision to be made is whether to employ a planar or three-dimensional (3D) cavity. In a 3D cavity, the field-sensitive qubit can be spatially separated from the small bias field required to saturate the magnetic sample. Moreover, such systems were already used to demonstrate a range of important results in the context of quantum measurements on magnon systems [40,41]. However, 3D cavities are typically much larger $(\sim \mathrm{cm})$ than the magnet $(\sim 100 \mu \mathrm{m})$, implying a bad mode matching and a fundamental limit on the achievable coupling (we require $\sim 100 \mathrm{MHz}$ ). Conversely, with a planar (quasi-one-dimensional) geometry the system has the advantage that the microwave mode is strongly confined to a volume that can be as much as 6 orders of magnitude smaller than in the 3D case $\left(\lambda d^{2} \sim 1 \mathrm{~cm} \times 1 \mu \mathrm{m} \times 1 \mu \mathrm{m} \sim\right.$ $10^{-6} \lambda^{3}$, where $d$ is the resonator width), although separating the bias field from the qubit is difficult. Accordingly, we suggest that an elegant way to herald the cat state would be to use the now classic methodology first proposed by Schuster et al. [65]. A relatively simple microwave quantum circuit could be constructed in which the YIG ellipsoid sits in the dielectric gap of a planar superconducting resonator coupled to a judiciously positioned transmon qubit. Spectroscopy would be performed on the system and the herald photon number measured via the occupancy-dependent Stark shift of the qubit.

The cat state can dissipate into the impurities in the YIG sample and into the cavity, with a total rate $\kappa_{\text {eff }}=\kappa_{m}+$ $g^{2} \kappa_{a} / \omega_{a}^{2}$, where $\kappa_{m}$ and $\kappa_{a}$ are linewidths of magnons and photons, respectively. For YIG, $\kappa_{m} \sim 10^{-4} \omega_{m}=2 \pi \times 10 \mathrm{kHz}$ [66]. We require $g / \omega_{a} \sim 0.1$ (see Fig. 2), and expect $\kappa_{a} \sim$ $2 \pi \times 10 \mathrm{MHz}$ [24,25], giving $\kappa_{\text {eff }} \sim 2 \pi \times 100 \mathrm{kHz}$ and a lifetime of $2 \pi / \kappa_{\text {eff }} \sim 10 \mu \mathrm{s}$. 
Conclusions. We proposed a setup to generate a magnetization cat state in a spin-microwave hybrid system. The scheme relies on adding a quanta to a squeezed vacuum of magnetization that is realized as the ground state of an anisotropic magnet [46]. We showed that cat states with the two components differing by $r_{\text {cat }} \hbar \sim 5 \hbar$ can be generated in sub-mm YIG samples, comfortably within the precision range of current quantum measurements of magnetization $[40,41]$. The size of the cat state is larger when the cavity is measured to be in higher photon numbers [Fig. 1 in Ref. [60]], although the heralding probabilities are much smaller. The lifetime of the cat states is $\sim 10 \mu$ s dominated mainly by indirect dissipation via cavity. Our analysis is valid when the system is in its ground state giving experimentally feasible temperature restrictions $T<5 \mathrm{mK}$. This setup could be adapted for generating nonclassical states of the magnetization asymptotically via feedback control over continuous optical measurement [67-70]. We envision our results to expand the field of quantum magnonics and applications of ferromagnets as quantum transducers and ultrasensitive magnetic field sensors, and to pave the way for protocols involving truly nonclassical macroscopic states of magnetization.

Acknowledgments. We thank J. Haigh for valuable discussion. S.S., V.A.S.V.B., and S.V.K. acknowledge financial support from the Max Planck Society.
[1] N. Ofek, A. Petrenko, R. Heeres, P. Reinhold, Z. Leghtas, B. Vlastakis, Y. Liu, L. Frunzio, S. M. Girvin, L. Jiang, M. Mirrahimi, M. H. Devoret, and R. J. Schoelkopf, Nature 536, 441 (2016).

[2] H. Jeong and M. S. Kim, Phys. Rev. A 65, 042305 (2002).

[3] T. C. Ralph, A. Gilchrist, G. J. Milburn, W. J. Munro, and S. Glancy, Phys. Rev. A 68, 042319 (2003).

[4] M. Mirrahimi, Z. Leghtas, V. V. Albert, S. Touzard, R. J. Schoelkopf, L. Jiang, and M. H. Devoret, New J. Phys. 16, 045014 (2014).

[5] W. J. Munro, K. Nemoto, G. J. Milburn, and S. L. Braunstein, Phys. Rev. A 66, 023819 (2002).

[6] T. C. Ralph, Phys. Rev. A 65, 042313 (2002).

[7] J. Huang, X. Qin, H. Zhong, Y. Ke, and C. Lee, Sci. Rep. 5, 17894 (2015).

[8] P. A. Knott, T. J. Proctor, A. J. Hayes, J. P. Cooling, and J. A. Dunningham, Phys. Rev. A 93, 033859 (2016).

[9] K. Huang, H. Le Jeannic, J. Ruaudel, V. B. Verma, M. D. Shaw, F. Marsili, S. W. Nam, E. Wu, H. Zeng, Y.-C. Jeong, R. Filip, O. Morin, and J. Laurat, Phys. Rev. Lett. 115, 023602 (2015).

[10] A. P. Lund, H. Jeong, T. C. Ralph, and M. S. Kim, Phys. Rev. A 70, 020101(R) (2004).

[11] J. Wenger, R. Tualle-Brouri, and P. Grangier, Phys. Rev. Lett. 92, 153601 (2004).

[12] B. Vlastakis, G. Kirchmair, Z. Leghtas, S. E. Nigg, L. Frunzio, S. M. Girvin, M. Mirrahimi, M. H. Devoret, and R. J. Schoelkopf, Science 342, 607 (2013).

[13] R. McConnell, H. Zhang, J. Hu, S. Ćuk, and V. Vuletić, Nature 519, 439 (2015).

[14] M. Aspelmeyer, T. J. Kippenberg, and F. Marquardt, Rev. Mod. Phys. 86, 1391 (2014).

[15] S. Hong, R. Riedinger, I. Marinković, A. Wallucks, S. G. Hofer, R. A. Norte, M. Aspelmeyer, and S. Gröblacher, Science 358, 203 (2017).

[16] R. Riedinger, A. Wallucks, I. Marinković, C. Löschnauer, M. Aspelmeyer, S. Hong, and S. Gröblacher, Nature 556, 473 (2018).

[17] F. Tebbenjohanns, M. Frimmer, V. Jain, D. Windey, and L. Novotny, Phys. Rev. Lett. 124, 013603 (2020).

[18] U. Delić, M. Reisenbauer, K. Dare, D. Grass, V. Vuletić, N. Kiesel, and M. Aspelmeyer, Science 367, 892 (2020).
[19] I. Shomroni, L. Qiu, and T. J. Kippenberg, Phys. Rev. A 101, 033812 (2020).

[20] H. Zhan, G. Li, and H. Tan, Phys. Rev. A 101, 063834 (2020).

[21] D. Lachance-Quirion, Y. Tabuchi, A. Gloppe, K. Usami, and Y. Nakamura, Appl. Phys. Express 12, 070101 (2019).

[22] V. Cherepanov, I. Kolokolov, and V. L'vov, Phys. Rep. 229, 81 (1993).

[23] Ö. O. Soykal and M. E. Flatté, Phys. Rev. Lett. 104, 077202 (2010).

[24] X. Zhang, C.-L. Zou, L. Jiang, and H. X. Tang, Phys. Rev. Lett. 113, 156401 (2014).

[25] Y. Tabuchi, S. Ishino, T. Ishikawa, R. Yamazaki, K. Usami, and Y. Nakamura, Phys. Rev. Lett. 113, 083603 (2014).

[26] Y. Tabuchi, S. Ishino, A. Noguchi, T. Ishikawa, R. Yamazaki, K. Usami, and Y. Nakamura, C. R. Phys. 17, 729 (2016).

[27] J. A. Haigh, A. Nunnenkamp, A. J. Ramsay, and A. J. Ferguson, Phys. Rev. Lett. 117, 133602 (2016).

[28] A. Osada, R. Hisatomi, A. Noguchi, Y. Tabuchi, R. Yamazaki, K. Usami, M. Sadgrove, R. Yalla, M. Nomura, and Y. Nakamura, Phys. Rev. Lett. 116, 223601 (2016).

[29] X. Zhang, N. Zhu, C.-L. Zou, and H. X. Tang, Phys. Rev. Lett. 117, 123605 (2016).

[30] S. Viola Kusminskiy, H. X. Tang, and F. Marquardt, Phys. Rev. A 94, 033821 (2016).

[31] X. Zhang, C.-L. Zou, L. Jiang, and H. X. Tang, Sci. Adv. 2, e1501286 (2016).

[32] C. Gonzalez-Ballestero, J. Gieseler, and O. Romero-Isart, Phys. Rev. Lett. 124, 093602 (2020).

[33] B. Heinrich, Y. Tserkovnyak, G. Woltersdorf, A. Brataas, R. Urban, and G. E. W. Bauer, Phys. Rev. Lett. 90, 187601 (2003).

[34] Y. Li, W. Cao, V. P. Amin, Z. Zhang, J. Gibbons, J. Sklenar, J. Pearson, P. M. Haney, M. D. Stiles, W. E. Bailey, V. Novosad, A. Hoffmann, and W. Zhang, Phys. Rev. Lett. 124, 117202 (2020).

[35] G. Woltersdorf, O. Mosendz, B. Heinrich, and C. H. Back, Phys. Rev. Lett. 99, 246603 (2007).

[36] R. Hisatomi, A. Osada, Y. Tabuchi, T. Ishikawa, A. Noguchi, R. Yamazaki, K. Usami, and Y. Nakamura, Phys. Rev. B 93, 174427 (2016).

[37] N. Zhu, X. Zhang, X. Han, C.-L. Zou, C. Zhong, C.-H. Wang, L. Jiang, and H. X. Tang, arXiv:2005.06429 [cond-mat.mes-hall]. 
[38] S. O. Demokritov, V. E. Demidov, O. Dzyapko, G. A. Melkov, A. A. Serga, B. Hillebrands, and A. N. Slavin, Nature 443, 430 (2006).

[39] S. M. Rezende, Phys. Rev. B 79, 174411 (2009).

[40] D. Lachance-Quirion, Y. Tabuchi, S. Ishino, A. Noguchi, T. Ishikawa, R. Yamazaki, and Y. Nakamura, Sci. Adv. 3, e1603150 (2017).

[41] D. Lachance-Quirion, S. P. Wolski, Y. Tabuchi, S. Kono, K. Usami, and Y. Nakamura, Science 367, 425 (2020).

[42] V. A. S. V. Bittencourt, V. Feulner, and S. V. Kusminskiy, Phys. Rev. A 100, 013810 (2019).

[43] M. Elyasi, Y. M. Blanter, and G. E. W. Bauer, Phys. Rev. B 101, 054402 (2020).

[44] J. Li, S.-Y. Zhu, and G. S. Agarwal, Phys. Rev. Lett. 121, 203601 (2018).

[45] J. Li and S. Gröblacher, Quantum Sci. Technol. 6, 024005 (2021).

[46] A. Kamra and W. Belzig, Phys. Rev. Lett. 116, 146601 (2016).

[47] A. Kamra, W. Belzig, and A. Brataas, Appl. Phys. Lett. 117, 090501 (2020).

[48] M. Dakna, T. Anhut, T. Opatrný, L. Knöll, and D.-G. Welsch, Phys. Rev. A 55, 3184 (1997).

[49] A. Ourjoumtsev, R. Tualle-Brouri, J. Laurat, and P. Grangier, Science 312, 83 (2006).

[50] B. R. Johnson, M. D. Reed, A. A. Houck, D. I. Schuster, L. S. Bishop, E. Ginossar, J. M. Gambetta, L. DiCarlo, L. Frunzio, S. M. Girvin, and R. J. Schoelkopf, Nat. Phys. 6, 663 (2010).

[51] G. Nogues, A. Rauschenbeutel, S. Osnaghi, M. Brune, J. M. Raimond, and S. Haroche, Nature 400, 239 (1999).

[52] L. Sun, A. Petrenko, Z. Leghtas, B. Vlastakis, G. Kirchmair, K. M. Sliwa, A. Narla, M. Hatridge, S. Shankar, J. Blumoff, L. Frunzio, M. Mirrahimi, M. H. Devoret, and R. J. Schoelkopf, Nature 511, 444 (2014).

[53] D. D. Stancil and A. Prabhakar, Spin Waves: Theory and Applications (Springer, New York, 2009).

[54] J. A. Osborn, Phys. Rev. 67, 351 (1945).

[55] T. Holstein and H. Primakoff, Phys. Rev. 58, 1098 (1940).

[56] D. Walls and G. Milburn, Quantum Optics (Springer, Berlin, 2008).

[57] R. J. Glauber, Phys. Rev. 131, 2766 (1963).
[58] J. Bourhill, N. Kostylev, M. Goryachev, D. L. Creedon, and M. E. Tobar, Phys. Rev. B 93, 144420 (2016).

[59] C. A. Potts and J. P. Davis, Appl. Phys. Lett. 116, 263503 (2020).

[60] See Supplemental Material at http://link.aps.org/supplemental/ 10.1103/PhysRevB.103.L100403 for detailed derivations of the ground state of the system and the heralded magnetization state after a single photon or a parity measurement which includes Refs. [56,71-76].

[61] We note that the cat size seems to be nonzero when $R \approx 0$, but this is merely an artifact of the definition which is irrelevant for $r_{\text {cat }} \sim 5$.

[62] R. Jozsa, J. Mod. Opt. 41, 2315 (1994).

[63] A. Uhlmann, Rep. Math. Phys. 9, 273 (1976).

[64] P. Andrich, C. F. de las Casas, X. Liu, H. L. Bretscher, J. R. Berman, F. J. Heremans, P. F. Nealey, and D. D. Awschalom, npj Quantum Inf. 3, 28 (2017).

[65] D. I. Schuster, A. A. Houck, J. A. Schreier, A. Wallraff, J. M. Gambetta, A. Blais, L. Frunzio, J. Majer, B. Johnson, M. H. Devoret, S. M. Girvin, and R. J. Schoelkopf, Nature 445, 515 (2007).

[66] S. Klingler, H. Maier-Flaig, C. Dubs, O. Surzhenko, R. Gross, H. Huebl, S. T. B. Goennenwein, and M. Weiler, Appl. Phys. Lett. 110, 092409 (2017).

[67] M. Yanagisawa, Phys. Rev. Lett. 103, 203601 (2009).

[68] K. Jacobs, L. Tian, and J. Finn, Phys. Rev. Lett. 102, 057208 (2009).

[69] K. Jacobs, J. Finn, and S. Vinjanampathy, Phys. Rev. A 83, 041801(R) (2011).

[70] J. Zhang, Y. xi Liu, R.-B. Wu, K. Jacobs, and F. Nori, Phys. Rep. 679, 1 (2017).

[71] J. Dereziński, J. Math. Phys. 58, 121101 (2017).

[72] J. Colpa, Physica A 93, 327 (1978).

[73] S. L. Braunstein, Phys. Rev. A 71, 055801 (2005).

[74] C. Navarrete-Benlloch, An Introduction to the Formalism of Quantum Information with Continuous Variables (Morgan \& Claypool, London, 2015), pp. 2053-2571.

[75] A. Royer, Phys. Rev. A 15, 449 (1977).

[76] W. P. Schleich, Quantum Optics in Phase Space (John Wiley \& Sons, New York, 2005), Chap. 3, pp. 67-98. 